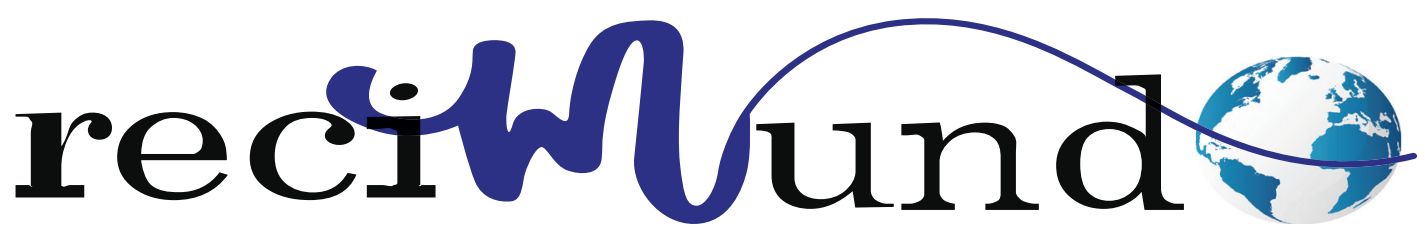

Revista Científica Mundo de la Investigación y el Conocimiento

DOI: 10.26820/recimundo/5.(2).julio.2021.205-219

URL: https://www.recimundo.com/index.php/es/article/view/1246

EDITORIAL: Saberes del Conocimiento

REVISTA: RECIMUNDO

ISSN: 2588-073X

TIPO DE INVESTIGACIÓN: Artículo de revisión

CÓDIGO UNESCO: 32 Ciencias Médicas

PAGINAS: $205-219$

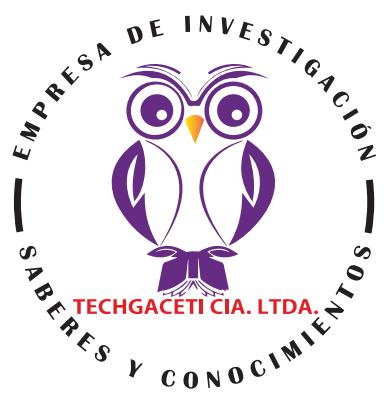

\title{
Síndrome burnout en docentes universitarios
}

Burnout syndrome in university teachers

Síndrome de burnout em professores universitários

Ingrid Esmeralda Gurumendi España'; Amelia Patricia Panunzio; Marco Antonio Calle Gómez³;

Maritza Alexandra Borja Santillán ${ }^{4}$

RECIBIDO: 11/04/2021 ACEPTADO: 15/06/2021 PUBLICADO: 30/07/2021

1. Magister en Gerencia y Administración en Salud; Medico; Universidad de Guayaquil; Guayaquil, Ecuador; ingrid.grumendie@ug.edu.ec; (D) https://orcid.org/0000-0001-6692-719X

2. Universidad del Zulia Facultad de Medicina División de Estudios para Graduados Doctorado en Ciencias de la Salud; Venezuela, patrypan@hotmail.com; (iD https://orcid.org/0000-0001-6242-5774

3. Magister en Derecho Civil y Procesal Civil; Especialista en Cirugía General; Especialista en Anestesiología; Diploma Superior en Docencia Universitaria; Doctor en Medicina y Cirugía; Abogado de los Tribunales y Juzgados de la Republica; Docente Facultad de Ciencias Médicas Universidad de Guayaquil, Guayaquil, Ecuador; (D) https://orcid.org/0000-00022706-1554

4. Doctora en Educación; Magister en Gerencia de Salud para el Desarrollo Local; Especialista en Gerencia y Planificación Estratégica en Salud; Diploma Superior en Desarrollo Local y Salud; Magister en Emergencias Médicas; Medico; Licencia-da en Ciencias de la Educación mención Lengua Inglesa y Lingüística; Docente Universidad de Guayaquil, Facultad de Ciencias Médicas, Guayaquil, Ecuador; (D) https://orcid.org/0000-0002-2803-4662

CORRESPONDENCIA

Ingrid Esmeralda Gurumendi España

ingrid.grumendie@ug.edu.ec

Guayaquil, Ecuador 


\section{RESUMEN}

El síndrome de Burnout o de "quemado en el trabajo", es una enfermedad laboral, reconocida por la Organización Mundial de la Salud como una de las más peligrosas que afecta a los profesionales y trabajadores, especialmente aquellos que prestan servicios de atención al público en el campo de la salud, la educación y la vigilancia de los comportamientos: médicos, enfermeras, docentes de los diferentes niveles educativos, trabajadores sociales, entrenadores deportivos, entre otros. En su etiología se identifica altas cargas de estrés no asimiladas, debidos a una gran presión de trabajo. Sus síntomas comprenden agotamiento emocional pérdida de sentido de las funciones del trabajo, incapacidad de comunicación y asociación, así como consecuencias en el sistema endocrino y cardiovascular, y su asociación con la diabetes y la obesidad. Uno de los segmentos profesionales más afectados por este síndrome de Burnout, es el de los profesores universitarios. Este artículo realiza un arqueo bibliográfico y documental para identificar los principales efectos de esta dolencia laboral en el personal docente de las universidades de Ecuador y de todo el mundo.

Palabras clave: Síndrome burnout, estrés, personal docente universitario.

\section{ABSTRACT}

Burnout syndrome or "burned at work" is an occupational disease, recognized by the World Health Organization, as one of the most dangerous that affects professionals and workers, especially those who provide care services to the public. in the field of health, education and monitoring of behaviors: doctors, nurses, teachers of different educational levels, social workers, sports coaches, among others. In its etiology, high unassimilated stress loads are identified, due to great work pressure. Its symptoms include emotional exhaustion, loss of sense of job functions, inability to communicate and associate, as well as consequences on the endocrine and cardiovascular systems, and its association with diabetes and obesity. One of the professional segments most affected by this burnout syndrome is that of university professors. This article carries out a bibliographic and documentary archeology to identify the main effects of this occupational disease on the teaching staff of the universities of Ecuador and around the world.

Keywords: Burnout syndrome, stress, university teaching staff.

\section{RESUMO}

A síndrome de burnout ou "queimado no trabalho" é uma doença ocupacional, reconhecida pela Organização Mundial da Saúde, como uma das mais perigosas que atinge profissionais e trabalhadores, principalmente aqueles que prestam serviços de atendimento à população. acompanhamento de comportamentos: médicos, enfermeiros, professores de diferentes níveis de ensino, assistentes sociais, treinadores desportivos, entre outros. Em sua etiologia, são identificadas altas cargas de estresse não assimiladas, devido à grande pressão de trabalho. Seus sintomas incluem exaustão emocional, perda do senso de funções no trabalho, incapacidade de comunicação e associação, além de consequências nos sistemas endócrino e cardiovascular e sua associação com diabetes e obesidade. Um dos segmentos profissionais mais afetados por essa síndrome de burnout é o de professores universitários. Este artigo realiza uma arqueologia bibliográfica e documental para identificar os principais efeitos desta doença ocupacional no corpo docente de universidades do Equador e do mundo.

Palavras-chave: Síndrome de burnout, estresse, professores universitários. 


\section{Introducción}

El síndrome de Burnout ya era reconocido desde 2010 por la Organización Mundial de la Salud como una de las enfermedades laborales más importantes en el mundo. En 2020, dado el auge de la dolencia y sus efectos sobre los trabajadores, el síndrome entró en la lista general de la OMS, como una de las dolencias propias de la actividad de algunas profesiones específicas, aquellas que prestan servicios o cuidados a otros seres humanos. De esta manera, el síndrome Burnout ha adquirido una relevancia significativa a nivel global (Morales \& Hidalgo Murillo, 2015).

El síndrome burnout ataca sobre todo a las profesiones que brindan servicios específicos a un público determinado. Por ello, ha sido establecida su prevalencia en los profesionales relacionados con la salud, tales como médicos y enfermeras. Pero también se ha venido investigando acerca de su impacto en la población de docentes de los diferentes niveles educativos, así como en entrenadores deportivos, en custodios de instituciones de corrección o penitenciarias, cuidadores de personas de la tercera edad, trabajadores sociales, administradores, policías y militares (Carlotto \& Goncalves Camara, Preditores de sindrome burnout em professores, 2007).

Los síntomas más notables del síndrome burnout son el agotamiento emocional, la despersonalización, la pérdida de sentido de su trabajo e incluso de la vida, y el colapso de la capacidad de comunicarse con los compañeros de labor y, en general, las personas a su alrededor. El síndrome burnout combina signos de otros malestares psicológicos de los profesionales y trabajadores, tales como la depresión, el estrés extremo, el agotamiento y las crisis existenciales, y configurando cuadros de extrema extenuación y derrumbe emocional y físico que exigen respuestas de todo tipo por parte de las instituciones donde laboran los afectados y todo su entorno (Mouzo Quintans, 2019).

El síndrome "burnout" significa "quemado" en inglés, pero ha recibido otras denominaciones: "consumido por el trabajo", "quemado por el trabajo", etc., y se halla estructurado en un conjunto de síntomas y de sufrimientos, conjuga síntomas físicos y psíquicos: fuertes jaquecas, dolores estomacales y problemas intestinales; un profundo agotamiento emocional, un derrumbe psicológico que echa por tierra las capacidades y la propia valía ante las tareas cotidianas más simples. El cansancio y la frustración derrumban al individuo en una postración brutal que aniquila sus capacidades básicas, haciéndolo incapaz de asumir responsabilidades y aislándolo de las personas que lo rodean cotidianamente. En el presente artículo, se focalizará la atención en la prevalencia del mencionado síndrome en un sector profesional específico: el de los docentes universitarios.

Ha sido constatado que los docentes son un segmento profesional muy expuesto a intensos y diversos estresores ocupacionales, los cuales conducen en muchos casos a contraer el síndrome Burnout. De hecho, la enfermedad se ha convertido en un fenómeno de dimensiones y carácter epidémico en esta categoría profesional. Otro aspecto significativo es que, aun cuando los docentes forman un grupo heterogéneo, pues en el interior de esa población existen distintas categorías, dependiendo del entorno donde actúan y de las instituciones donde laboran, sean escuelas, universidades o institutos (Carlotto \& Goncalves Camara, Preditores de sindrome burnout em professores, 2007).

En este artículo se propone recopilar, registrar, sistematizar los datos, conceptos y explicaciones relevantes sobre la prevalencia del síndrome de burnout sobre los docentres universitarios, se realizará una revisión bibliográfica y documental. 


\section{Metodología}

Para establecer el estado de la cuestión acerca de la prevalencia del síndrome de Burnout en distintas profesiones, y específicamente en el personal docente universitario, se realizará una revisión crítica de artículos científicos relativos a estudios de la presentación de esta enfermedad en el mundo, en América Latina, en instituciones educativas de los diversos niveles. Esa revisión se avanzará mediante una contextualización histórica y situacional, que permitirá realizar la vinculación entre las temáticas de los textos, en los cuales se identificará y precisarán los conceptos clave (Sierra Bravo, 1994).

En el presente artículo se realizó una revisión documental a partir de artículos científicos y documentos de organizaciones internacionales, en publicaciones acerca de la prevalencia del síndrome de Burnout, su relevancia como enfermedad laboral en las diferentes profesionales, así como su caracterización a través de las teorías que se han desarrollado para su diagnóstico y tratamiento.

Para realizar la investigación fue fundamental detectar, obtener y consultar documentos y bibliografía pertinente al problema de investigación, así como la extracción y recopilación de información de interés. Al recopilar la información de referencias fue posible extraer datos, eventos, opiniones, resultados, explicaciones, que nos permitieron dotarnos de una teoría, que sirvió para describir, explicar e interpretar los fenómenos que hemos delimitado como objeto de investigación (Hernández Sampieri, 2014.).

\section{Resultados}

De la revisión bibliográfica y documental se obtuvieron los siguientes datos, conceptos y explicaciones de estudios acerca del síndrome de burnout, su descubrimiento y caracterización, su reconocimiento por los organismos internacionales de la salud, su manifestación en los distintos segmentos profesionales, especialmente en los trabajadores de la salud, en docentes y otras profesionales dedicados al cuidado o la vigilancia, como es el caso de los trabajadores sociales, policías y entrenadores deportivos.

El burnout o síndrome de estar quemado, o de quedarse exhausto, como respuesta a la excesiva exigencia laboral en profesiones de servicio, es una enfermedad descrita en el índice internacional de la Organización Mundial de la Salud, como problema relacionado con el desgaste profesional, que se caracteriza por su efecto multidimensional, que va más allá del cansancio habitual, comprometiendo a la esfera fisiológica, física y social; en esto se describen alteraciones en el sueño, afecciones cardiovasculares, endócrinas, gastrointestinales, dolores musculares, cefalea, pérdida de peso, alteraciones inmunológicas, alteraciones en el ciclo menstrual y daño a nivel del encéfalo. El ambiente laboral se ve seriamente afectado, la productividad decrece, la calidad del trabajo también, el ausentismo, y lo más preocupante, la actitud negativa del enfermo hacia las personas a quien presta el servicio (Gil Monte, 2011).

El síndrome fue enteramente descrito por los investigadores norteamericanos Maslach y Jackson en la década de los ochenta del siglo XX, y establecieron que el Burnout es ocasionado por el estrés laboral crónico, que afecta las actitudes y los sentimientos hasta tornarlos negativos hacia el ambiente laboral, además de provocar una serie de síntomas que influyen negativamente en el conjunto de los afectos y las emociones de individuo, así como sobre las actitudes, las cogniciones, la conducta y en el sistema fisiológico del trabajador. Así, la doctora Maslach concluye que el burnout se configura como "un síndrome tridimensional caracterizado por agotamiento emocional, donde los individuos se encuentran emo- 
cionalmente agotados y sobrepasados en cuanto a sus recursos emocionales, la despersonalización que implica actitudes negativas, cínicas e impersonales y la reducida realización personal con disminución de los sentimientos de competencia y éxito en el trabajo, autoevaluándose negativamente" (Olivares, 2017).

A lo largo de las seis últimas décadas, se ha buscado otorgar un término específico y brindar de esta manera una correlación clínica-social a la situación descrita actualmente, evidenciándolo en diferentes trabajos como los publicados por Schwartz y Will en 1953, en el que describían un sentimiento de baja moral y distanciamiento de los pacientes experimentado por una parte de las enfermeras de un servicio de psiquiatría (Olivares, 2017).

En 1961, Graham Greene autor de novelas llama por primera vez al síndrome de desgaste profesional, en una cita como: "a burnout case". Sin embargo, el llamarlo Síndrome de Burnout es desde el año 1974, cuando en Estados Unidos de Norteamérica el psiquiatra Herbert Freudenberg, fue el primero que acuñó el término de "burnout" en el sentido de "agotamiento profesional", definiéndolo como "la extinción de la motivación o el incentivo, especialmente allí donde la dedicación a un objetivo o a una relación fracasa en conseguir los resultados deseados". Y se refirió así de la afección de los trabajadores de una clínica de toxicómanos que llegaban con gran entusiasmo al trabajo pero que al pasar el tiempo estaban desmotivados, sin ánimo y con evitación a los usuarios y actitudes negativas, definiéndolo así, en una forma clínica (Olivares, 2017).

Lo referido por Maslach en uno de sus análisis explica, en las personas para lo que al inicio era un trabajo importante, representativo y retador, se torna frío, insatisfactorio y absurdo; cabe recalcar que el agotamiento ocurre en profesiones que tratan con servicios humanos, y la enseñanza ha sido considerada como una profesión que ha reportado altos niveles de agotamiento (Lou \& Chen, 2016).

Una de las autoras más citadas en el tema del burnout es la doctora Maslach (1976), una psicóloga social que estudiaba las respuestas emocionales de las personas que realizaban trabajos de ayuda, categorizando el cuadro como "Burnout" en aquellos que habían perdido el sentido de la responsabilidad y se comportaban cínicamente en su trabajo. El término tuvo mucho éxito, porque de alguna forma todo el mundo entiende lo que quiere decir sin necesidad de muchas explicaciones y además no implica ninguna etiqueta peyorativa a priori, como podrían haber tenido otros términos empleados por psicólogos o psiquiatras. Según Christine Maslach y Andrea Jackson, quienes fueron consideradas como las pioneras del burnout, lo configuran como un síndrome tridimensional caracterizado por:

- Agotamiento emocional, que es el elemento clave del síndrome, y se refiere a que los individuos presentan sentimientos de estar emocionalmente agotados y sobrepasados en cuanto a sus recursos emocionales.

- Sensaciones de sobreesfuerzo, despersonalización con actitudes de insensibilidad: que implica actitudes negativas, cínicas e impersonales, generándose sentimientos demasiado distantes hacia otras personas.

- Baja realización personal con sentimientos de incompetencia profesional y falta de éxito en el trabajo. Así, como una tendencia a evaluarse negativamente a sí mismo, particularmente en el trabajo con otras personas (Olivares, 2017).

Estas autoras son las que posteriormente diseñaron el cuestionario más conocido y empleado de los que se utilizan para medir y valorar el burnout en diferentes colectivos, que se conoce como el Maslach Burnout Inventory. Por otra parte, la inteligencia

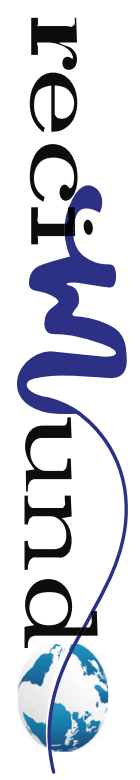


emocional ha sido observada como una estrategia de regulación para el Burnout, que estaría conformada por habilidades para percibir, asimilar, comprender y regular emociones, evaluando así tres componentes: reparación, claridad y atención emocional.

El Maslach Burnout Inventory (MBI) de Maslach y Jackson, es un instrumento, creado en 1981 y modificado en 1986, que contiene 22 ítems dirigido a profesionales de servicio, que valora una escala de seis grados y tres dimensiones que definen el burnout: Agotamiento Emocional (AE), Despersonalización (DP) y baja Realización Personal en el Trabajo (RPT). Inicialmente constaba de dos partes, una de frecuencia y otra de intensidad, las respuestas estaban enfocadas a sentimientos y pensamientos relacionados con el ámbito y su desempeño habitual en el trabajo. En 1981 incorporaba 25 ítems distribuidos en cuatro escalas, las tres antes expuestas y otra "Implicación con el trabajo"; pero en la versión de 1986 fue eliminada, posterior a ello se estableció con 22 ítems, manteniendo así, las mismas dimensiones distribuidas, al agotamiento emocional (9 ítems), despersonalización (5 ítems) y realización personal en el trabajo (8 ítems), estimando una puntuación baja, media o alta. (Alvarez Gallego \& Fernández Ríos, 2002).

Existen algunos cuestionarios dirigidos a grupos específicos como: el MBI-Human Services Survey (MBI-HSS), dirigido a profesionales de servicios humanos, también se tiene el MBI-Educators (MBI-ES), dirigidos a profesionales de la educación. Y, finalmente, el MBI-General Survey (MBI-GS), una nueva versión del MBI (Gil Monte, 2011).

Es necesario tomar en cuenta a los factores ambientales y psicológicos como causa de una conducta anómala en la alimentación, que conllevan a un aporte energético excesivo en ausencia de actividad física como parte del sedentarismo.
Siendo el burnout la respuesta del individuo a todos los estresores crónicos que pueden darse en el ambiente laboral, es necesario conocer la avasalladora sintomatología que provoca, no sólo en el ámbito laboral, como un agotamiento inmedible, sensación de pereza y un decrecimiento o frustración de la realización personal, sino también a nivel cardiometabólico, músculo esquelético y mental (Pedro, 2001).

La OIT. Organización Internacional del Trabajo, mediante sus convenios y recomendaciones trata en sus artículos literales detallados sobre la Seguridad y salud de los trabajadores (Organización Internacional del Trabajo, 2020).

R164 - Recomendación sobre seguridad y salud de los trabajadores, 1981 actualizado 1983.

R197 - Recomendación sobre el marco promocional para la seguridad y salud en el trabajo, 2006

Art. 1. Literal e) el término salud, en relación con el trabajo, abarca no solamente la ausencia de afecciones o de enfermedad, sino también los elementos físicos y mentales que afectan a la salud y están directamente relacionados con la seguridad e higiene en el trabajo.

Contar con un sistema que abarque la buena gestión de la seguridad y la salud en el trabajo (SST), está considerada dentro de la Norma ISO 450001, para obtener un trabajo seguro y saludable, y lograr la prevención de los daños y del deterioro de la salud que están relacionados con el trabajo. Se enfoca directamente a:

Protección de los trabajadores - identificación de peligros y la gestión de riesgos contribuye a mantener un ambiente de trabajo más saludable y seguro, asi reducir el número de accidentes y los problemas de salud producidos en el lugar de trabajo. 
Reducción de los riesgos - reducir los riesgos, proteger a los trabajadores y controlar las amenazas en infraestructura que causan accidentes.

Cumplimiento legal - identificación de la legislación vigente y la implementación de los requisitos aplicables. Las organizaciones deben mantenerse conforme a la ley para de esa manera disminuir las quejas, pagar la seguridad social, evitar consecuencias financieras, y debilitar el estigma de la publicidad negativa.

Responsabilidad - cumpliendo y obteniendo la certificación, es una manera de demostrar la responsabilidad y compromiso en materia de Seguridad y Salud en el trabajo a sus empleados. Libro Fremap ISO 45001.

El Ministerio de Salud Pública (MSP), busca mantener y fomentar la buena salud de toda la comunidad. Mediante el Modelo de Atención Integral de Salud (MAIS) se logra mantener una misma forma de atención y dar continuidad considerando los determinantes de la salud, que tienen un potencial para generar protección o daño para la salud individual y colectiva. La protección de la salud depende de la capacidad de la sociedad para mejorar la interacción entre la actividad humana y el ambiente químico, físico y biológico. Esto debe hacerse de manera que promocione la salud humana y prevenga la enfermedad, manteniendo el equilibrio y la integridad de los ecosistemas, y evitando comprometer el bienestar de las futuras generaciones. Además este modelo plantea integrar la investigación, la formación y capacitación del talento humano, para fortalecer los procesos de investigación, formación y capacitación, para que la producción de conocimientos se constituya en una herramienta que contribuya al mejoramiento de las condiciones de salud y vida de la población.

Ley Orgánica de Educación también tiene su consideración en los siguientes artículos
(República del Ecuador, 2016)

Art. 9.- La educación superior y el buen vivir.- La educación superior es condición indispensable para la construcción del derecho del buen vivir, en el marco de la interculturalidad, del respeto a la diversidad y la convivencia armónica con la naturaleza.

Los educadores, como trabajadores que laboran en función al sistema educativo del estado, tienen sobre sí una gran responsabilidad, por lo que son objeto de una gran exigencia laboral. Esta presión laboral puede esas alteraciones de los parámetros cardiometabólicos, asociados al estrés y al síndrome Bournot. El síndrome Burnout es, además, un factor de riesgo en la agudización de enfermedades cardiometabólicas, así como la diabetes, las hiperlipidemias, la hipertensión, el aumento de masa corporal entre otras. Estas dolencias coaligadas al Burnout, son causantes de millones de muertes a nivel mundial. Así mismo, es importante la asociación entre el síndrome de burnout y la obesidad. En las revisiones bibliográficas revisadas, se señala que un $33 \%$ de las investigaciones realizadas hasta el año 2016, se ha constatado una correlación positiva entre el estrés laboral y el índice de masa corporal (Santana Cárdenas, 2016).

El estrés no solo provoca irregularidades de tipo gástrico o muscular, como frecuentemente se observaba, sino que también existe una alteración de los hábitos de alimentación provocado por el mismo estrés, que desencadena problemas relacionados con el peso. Hay investigaciones de la Organización Internacional del Trabajo, que comprueban el vínculo entre el aumento de los gastos de salud y costos laborales, y la presencia de estrés laboral y burnout, en los países de América Latina (Botero Álvarez, 2012).

Así mismo, llama la atención que, de doce artículos analizados, el 33\% de estudios

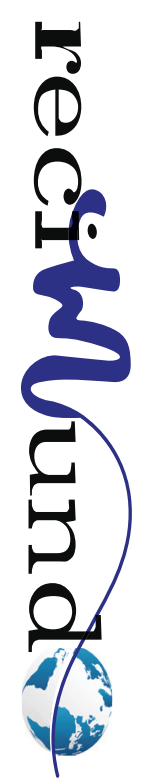


que fueron realizados en países desarrollados se relacionaban con estrés laboral y peso corporal. Al analizar que el estrés laboral no solo está directamente relacionado con burnout, sino también con otras afecciones del organismo, en este caso el peso corporal. Esto guarda relación con lo que la Organización Internacional del Trabajo (OIT) predice para el año 2030, una gran incapacidad laboral provocada por el estrés laboral (Santana Cárdenas, 2016).

En Latinoamérica, se han realizado importantes estudios de este síndrome, uno de ellos es una recopilación de 89 estudios de la década que va del año 2000 al 2010 de 12 países de la región, en éste, pese a que se describen iguales manifestaciones entre los que padecen la afección comparativamente hablando entre Latinoamérica y el resto del mundo, concluyen que en la mayoría de los estudios, no hubo una cavilación acerca de la tipicidad de los procesos de trabajo propios de la región.

En Argentina, la Comisión Nacional de Salud en el año 2008, determinó que la prevalencia de burnout en profesionales de la salud fue del $47.19 \%$ a nivel general, y al considerar criterios clínicos llegó al 12.55\%. En Chile, un estudio de la Universidad Metropolitana de Ciencias de la Educación reflejó en educadores, similar resultado que en Argentina dando $46.2 \%$ con altos niveles de agotamiento emocional. En otros países de la región se han realizado estudios en personal policial, en instituciones estatales de atención al público y también en la empresa (Díaz Bambulla \& Gómez, 2016).

Otro estudio acerca de la incidencia del síndrome en personal docente universitario, (Merces, Almeida Lopes, Souza e Silva, \& Souza Oliveira, 2017), se propuso como objetivo estudiar la vinculación entre el síndrome Burnout y las condiciones metabólicas en profesionales de enfermería y profesionales de la Atención Primaria de Salud
(APS), en Bahía Brazil. Para ello, se realizó un estudio piloto compuesto por 60 profesionales de enfermería que desarrollaban actividades asistenciales en la APS, siendo $38,3 \%$ enfermeras y $61,7 \%$ auxiliares y técnicas de enfermería. Se aplicó un cuestionario de secciones socio demográfico, laboral, de estilo de vida y biología humana. Evaluaron el SB utilizando el Maslach Burnout Inventory - Human Services Survey (MBI - HSS), de 22 ítems que exploran aspectos del nivel de agotamiento emocional (EE), despersonalización (DP) y reducida realización profesional (RRP), apuntando a un nivel alto en EE o DP, o nivel bajo en RRP de forma independiente, para ser considerado con SB. Las condiciones metabólicas, fueron evaluadas por cuestionarios autorreferidos, medidas de presión arterial, evaluaciones antropométricas, tales como: Circunferencia abdominal, peso y altura. Se realizó análisis de glicemia, HDL colesterol y triglicéridos, con técnicas de laboratorios enzimáticas y colorimétricas convencionales, previa firma del Consentimiento Informado, en donde se evidenció la alteración de muchos de ellos.

En Ecuador, el profesor que desde el año 2000 se sometía al cambio del modelo pedagógico tradicional por el constructivista, y que venía haciéndose secuencialmente, con la "Revolución Ciudadana" se ve abocado a un cambio extremo; esto indudablemente significó un gran impacto y afectación de su desempeño y salud mental.

Sabiendo que el burnout está relacionado directamente con las condiciones de excesiva exigencia de trabajo, los estamentos docentes incluidos en la Ley de Educación Superior, y en algunos casos una doble carga (añadida) de horas de trabajo de otra institución, pudieran conllevar al cansancio, astenia, factores directos asociados con este riesgo psicosocial (Cárdenas, Méndez, \& González, 2014). 
En el país, el estudio más actual en marcha corresponde al año 2017, de Marina del Rocío Ramírez, docente investigadora de la Universidad Técnica Particular de Loja (UTPL), habiendo reportado que la prevalencia del Síndrome de burnout en los profesionales de la Salud está alrededor del 30 por ciento; este estudio inició en la ciudad de Loja, pero se extendió a 18 provincias del Ecuador (Ramírez, 2017).

Al respecto, se percibe, un docente preocupado por cumplir con lo que la ley establece, el órgano ejecutor llamado Consejo de Educación Superior exige una revolución en la universidades, la Secretaria de Estado SENESCYT, vigila ese cumplimiento; con él llegan, cierre de universidades, exigencias de maestrías y doctorados, centros de excelencia, pero una evolución necesita de tiempo, no hay infraestructura adecuada, las plataformas virtuales tardan en llegar; si bien se dedican recursos económicos, la economía del estado es insuficiente, el fenómeno político sufre transformaciones, la sociedad exige una universidad de excelencia, y los docentes son los que reciben el peso de esa exigencia (Arquero \& Donoso, 2013); (Chavarría, Colunga, \& Peláez, 2017).

Hacer frente a todas estas demandas psicosociales, en el tiempo exigido, aunada a otros factores laborales, puede generar afección negativa en el proceso salud-enfermedad, que según el análisis de vida de docentes de manera individual, no sólo trae consecuencias mentales o psicológicas, sino también daños corporales orgánicos, como pueden ser afecciones hormonales, pues se produce una secreción anormal de cortisol, hormona que en cantidades elevadas puede desencadenar efectos que predisponen al trabajador a enfermedades crónicas degenerativas. Estos impactos sobre la salud del trabajador traen como consecuencia el aumento de los costos del sistema de salud y un impacto negativo en el bienestar de quienes desempeñan la docencia a nivel universitario.

La conducta alimentaria y obesidad, el burnout, el índice de masa corporal y la relación con el estrés han sido estudiados ampliamente (Santana Cárdenas, 2016). Estos hallazgos en la literatura científica mundial forman parte de la evidencia de la influencia psíquica y social del individuo, como desencadenante de trastornos cardiometabólicos que inciden en la salud.

Frente a la magnitud de las afecciones que provoca el burnout, y el incremento del número de afectados es importante plantear como problema la relación entre sobrepeso, obesidad y otros factores de riesgo en los docentes en el Ecuador. Los estudios realizados (Mora, Flores, Hernández, Sánchez, \& Marroquin, 2015); (Cabezas, 2016); (Ramírez, 2017), han demostrado la correlación de los casos de docentes afectados por el burnout y los diagnosticados con obesidad impuesta por la alteración de la segregación endocrina del cortisol. Esto, por otra parte, confirma los estudios realizados en otras naciones del mundo.

Así mismo, se conoce que existen estresores psicosociales determinantes del burnout en docentes universitarios, tal es el caso de los más importantes señalados en las investigaciones citadas y recabadas en la experiencia personal como, interacciones con los estudiantes que cada día se tornan más complejas por la indisciplina respaldada en los derechos que se expiden en los reglamentos; exceso de trabajos administrativos cumpliendo funciones de coordinador, coevaluador, auditor áulico; evaluación al desempeño docente, autoevaluación, heteroevaluación y también a los estudiantes, evaluaciones periódicas, exámenes de recuperación, exámenes de mejoramiento, exámenes atrasados; falta de incentivos, no convocatoria a concursos, requisitos inalcanzables para ascensos cuando los hay, control docente, y expectativa en si existirá o no la renovación de contratos o el fin de

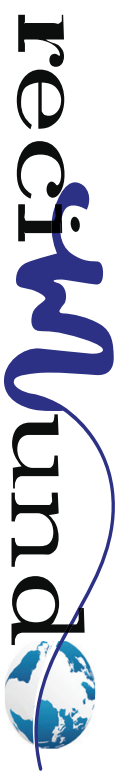


contratación por cumplimiento del tiempo máximo determinado por la ley (Caballero, Hederich, \& Palacio, 2010).

Estos estresores conducen al docente al cansancio, deshumanización, que se traduce en ver a los estudiantes como enemigos y sentimientos de odio a sus superiores que llevan lentamente a desórdenes en su personalidad, llegando a trastornos mentales y a la despersonalización, agravados aun con los trastornos cardiometabólicos propios de estos desórdenes como la hipertensión, diabetes, obesidad, pudiendo llegar hasta la muerte súbita, así lo menciona Ayuso (2006).

Existen evidencias, registradas en la literatura científica mundial, que señalan que la realidad del Ecuador, en cuanto a la asociación de burnout en docentes universitarios con obesidad y sobrepeso y secreción de cortisol, puede ser extrapolable a otras regiones del mundo. Otro aspecto estudiado es la posibilidad de que esto se asocie con otros factores psicosociales en el contexto ocupacional, para convertirse en determinantes de enfermedades cardiovasculares. En todo caso, en Ecuador todavía no han sido estudiadas suficientes evidencias de esta comorbilidad.

Lo que sí se ha constatado suficientemente es que los riesgos psicosociales se encuentran asociados con el estrés, y éste, a su vez, con el trabajo. Las presiones y demandas laborales sobre las personas son cada vez son mayores. Las exigencias propias de los sistemas, la presencia de incertidumbre, el no poder controlar los eventos, perder la capacidad de comunicarse, sentir que ya no se tienen los medios para enfrentar las dificultades y el temor ante situaciones nuevas y desconocidas, provocan en los individuos una gran vulnerabilidad, que, a pesar de poseer el conocimiento y habilidades necesarias, ponen en peligro su capacidad de resistencia (Pérez Jauregui, 2018).
Las altas exigencias en el trabajo contribuyen a ocasionar un desequilibrio en la salud física y mental, como lo muestran diversos estudios que, igualmente, constatan la comorbilidad del síndrome de burnout y las enfermedades crónico-degenerativas. Se confirma que el estrés laboral y el burnout están vinculados a enfermedades cardíacas, endócrinas, depresivas y trastornos musculo-esqueléticos, tanto en los países subdesarrollados como en aquellos que se hallan en los primeros lugares en avances tecnológicos (Mora, Flores, Hernández, Sánchez, \& Marroquin, 2015), (Santana Cárdenas, 2016) (Jonsdettir \& Dahlman Sjors, 2018) (Guyton \& Hall, 2016) (Rodríguez, bermello, Pinargote, \& Durán, 2018) (Serrano, 2006).

La aparición de casos de síndrome burnout entre los docentes universitarios, afecta el vínculo docente-estudiante y daña el proceso de educativo en su conjunto. Además, esta prevalencia del burnout en este tipo de personal genera ausentismo laboral, bajo nivel de aporte científico y metodológico. Esto, por supuesto, tiene consecuencias negativas en la formación profesional del país (Chávez, 2016) (Díaz, 2021).

Rozas Caamaño (2016), realizó un estudio comparativo de las dimensiones del desgaste profesional (burnout) en docentes que trabajan en las Facultades de Salud, Educación y Ciencias Sociales de la Universidad Santo Tomás. Los resultados indicaron que el $48 \%$ presentaron proclividad al burnout, el 62\% no se encontró afectado; el $55 \%$ se sentían satisfechos en su trabajo; el $59 \%$ presentó un bajo nivel de dificultades en las relaciones interpersonales y el $16 \%$ un alto nivel de agotamiento emocional. Sin embargo, el $99 \%$ se encuentra en los niveles medios o altos de degradación de la competencia profesional; el 53\% presentó un nivel de riesgo en despersonalización; el $58 \%$ de los docentes presentaron niveles medios y altos de trastornos físicos asociados al burnout; el 55\% trastornos conduc- 
tuales asociados al burnout. Al realizar el análisis comparativo, se evidenció diferencias de trastornos conductuales asociados a las facultades, siendo los docentes de la Facultad de Salud quienes presentaron mayores afecciones.

En la revisión de la literatura científica acerca de la prevalencia del síndrome burnout en el personal docente, se abordan los casos de diferentes instituciones de educación superior en varios países.

Así, Chavarría, Colunga, Loria y Peláez (2017) estudiaron el Síndrome burnout en médicos docentes de un hospital de tercer nivel en México, con el objetivo de identificar la presencia de burnout en el personal médico dedicado a labores docentes y los factores asociados a su presentación. De un total de 12 médicos de pregrado y 4 de posgrado se ubicaron con nivel alto en la escala de Burnout y además determinaron que las variables: especialidad, número de hijos, situación laboral, condicionales organizacionales y preocupaciones personales tienen mayor efecto en su presentación.

Rodríguez y colaboradores (2018) determinaron el impacto del estrés en la salud mental de los docentes de la Universidad Técnica de Manabí. Evaluando diez facultades: Filosofía, Ciencias Humanísticas, Ciencias Matemáticas, Agronomía, Ciencias Administrativas, mostrando datos relacionados a los trastornos del ánimo, que se consideran como indicadores los signos y síntomas asociados a nerviosismo, ansiedad, temor, ira, negatividad, datos que revelan que existe una tendencia de la influencia de estresores en el estado de ánimo que repercute en su salud mental y física; aspecto que se corrobora en el estudio realizado por (Ayuso, 2006), quienes encontraron que las causas del estrés son: la sobrecarga de trabajo, presión del tiempo, ambigüedad de roles, clima organizacional, inestabilidad laboral, problema de interrelación con el equipo multidisciplinario, lo que afecta en el desempeño laboral y disminuye la calidad de los cuidados y la productividad; aspecto que no se aleja de la realidad de los docentes universitarios. Se evidencian las reacciones psicosomáticas, de la sub-variable pseudoneurológico, que experimentan los docentes de la Universidad Técnica de Manabí.

\section{Discusión}

Como en el caso del personal de la salud, los docentes, profesores y maestros, desarrollan un trabajo que consiste en prestar un servicio y atender a unos usuarios de distintas edades.

Los exámenes mediante los protocolos generalmente aplicados para identificar y medir la dolencia (cuestionario MIB) han mostrado una alta prevalencia entre docentes universitarios.

Se ha observado en todo el mundo que los docentes a todo nivel se hallan expuestos a diversos estresores ocupacionales, los cuales los hacen vulnerables y predispuestos a sufrir el síndrome Burnout. Puede afirmarse que esta enfermedad es un fenómeno que ha adquirido dimensiones y carácter epidémico; aunque hay que tomar en cuenta que los docentes forman un grupo heterogéneo, pues en el interior de esa población existen distintas categorías con diferentes funciones, atribuciones, desafíos y recompensas, dependiendo del nivel del entorno donde actúan, asumiendo que los contextos sociales de las instituciones donde están insertos (escuelas, universidades, institutos), son diferentes.

Los docentes se hallan expuestos a estresores ocupacionales, que son aquellos aspectos del trabajo (exposición ante los estudiantes, exigencias de planificación, preparación y evaluación de la enseñanza, manejo de conflictos, etc.), que generan tensiones recurrentes y desequilibrios entre la percepción de las demandas de trabajo

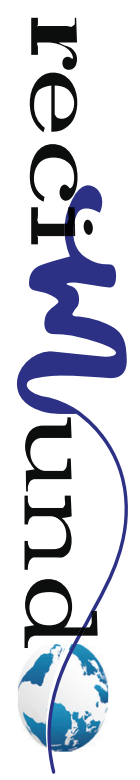


y su capacidad de tener los conocimientos y habilidades necesarias para cumplir con sus funciones. Al mismo tiempo, se presentan los estresores ocupacionales psicosociales, relacionados con la naturaleza del trabajo y sus relaciones interpersonales (Carlotto \& Camara Goncalves, 2017).

El síndrome de Burnout ocurre como una respuesta inadecuada y crónica a los estresores psicosociales presentes en el contexto del trabajo. En el caso de los docentes, se aprecian cuatro dimensiones de su ocurrencia:

- Destrucción de las ilusiones respecto al trabajo,

- Desgaste Psicológico,

- Indolencia y

- Culpa

Los estudios realizados en diversas instituciones educativas muestran que de las tres subescalas que se presentaron con mayor frecuencia son los factores del agotamiento emocional, la despersonalización y la falta de realización personal.

Otras características de la población docente se refieren a sus relaciones personales y familiares, conectados con su edad. Los que tenían menos tiempo trabajando como docentes también presentaron con mayor frecuencia síntomas del síndrome, así como el grupo que había trabajado en un lapso de 6 a 10 años, y contratados, en contraste con los docentes de mayor antigüedad. Los resultados, obtenidos por medio del análisis de regresión lineal apuntaron a las variables "autonomía", "apoyo social", "conflicto de papel" y "conflictos interpersonales" como principales predictores de Burnout (Pineda Valladares, Rivera Rodas, \& Ríos Verdugo, 2014).

En estos aspectos referidos a la satisfacción con el trabajo, pueden empezar a diferenciarse los docentes universitarios de lo no universitarios. Entre los universitarios, la variable de mayor valor explicativo es el de agotamiento emocional al mismo tiempo que la insatisfacción con el sueldo. Se notó que, a medida que aumenta su satisfacción con la remuneración, disminuye el sentimiento de desgaste. Esto puede interpretarse a la luz de la constatación general de que el profesor universitario, en comparación con los docentes de escuelas, es un profesional que tiene mayores ventajas, mejores salarios y condiciones de trabajo en general.

Esto sugiere que ese segmento profesional de profesores universitarios, al ser objeto de mayores inversiones en su formación científica, además de requerir experiencia profesional y titulaciones, puede crearse mayores expectativas, las cuales, al no ser atendidas, elevarían su grado de desgaste emocional. Es interesante destacar que esa situación se hace más presente entre los profesores más jóvenes quienes, probablemente, se crean mayores expectativas, lo cual se confirma cuando se cruza con la variable de la edad en este modelo de correlación.

Otra variable con poder explicativo del aspecto de la despersonalización, en profesores universitarios, fue la Carga Horaria dedicada a tareas de investigación. Según los resultados, la elevación de esta exigencia aumenta a su vez el sentimiento de distanciamiento respecto a los alumnos, es decir, los usuarios del servicio de la enseñanza. Algunas instituciones universitarias exigen hasta un límite de 8 horas semanales destinadas a la realización de esas actividades. Al mismo tiempo que realizan sus investigaciones, los docentes realizan asesorías y orientación a los alumnos en la confección de sus monografías, aunque generalmente con poca experiencia y conocimiento, aparte de una mayor identificación con tal actividad. Otra variable explicativa de relevancia se refiere al reconocimiento que obtiene el profesor por su labor de investigación, lo cual da pie a la hipótesis de que 
los profesores al realizar sus actividades de investigación, necesitan obtener de retorno, algún tipo de compensación por parte de la institución. El profesor universitario, al inicio de su carrera, busca, entre otros aspectos, el reconocimiento académico.

\section{Conclusiones}

1. El síndrome de burnout es una enfermedad laboral de gran prevalencia entre los docentes universitarios, por lo que se impone la toma de decisiones en relación a medidas de previsión y tratamiento mediante un adecuado apoyo social.

2. El síndrome burnout se presenta en los profesores universitarios con su sintomatología típica de agotamiento emocional, pérdida de sentido de la profesión, despersonalización e incapacidad de comunicación, aspectos que obstaculizan esencialmente la labor misma de la educación y tiene repercusiones en la calidad de vida del educador, así como en la del servicio educativo.

3. Aspectos como la edad, la remuneración, el estrés laboral, la relación con los estudiantes y el reconocimiento institucional son factores determinantes en la presentación de casos de burnout entre los profesores universitarios

4. Hay que dotar a los docentes universitarios de estrategias de afrontamiento a situaciones de estrés intenso para evitar la proliferación del síndrome de Burnout. Así mismo prever terapias y dinámicas de grupo para el debido tratamiento.

5. En Ecuador también se ha incrementado la cantidad de casos del síndrome burnout en las nuevas condiciones impuestas por situaciones advenidas, como la reciente pandemia del COVID 19, que abruma a los educadores con nuevas exigencias y nuevos roles que ameritan una formación adecuada para poder responder a los requerimientos novedosos de las actuales condiciones de desempeño profesional

\section{Bibliografía}

Alvarez Gallego, E., \& Fernández Ríos, L. (octubre de 2002). El síndrome de burnout o el desgaste profesional: revisión de estudios. Revista de la Asociación Española de Neuropsiquiatría, XI(39).

Arquero, J., \& Donoso, J. (2013). Docencia, investigación y Burnout: el síndrome del quemado en profesores unviersitarios de contabilidad. Revista de Contabilidad, 94-105.

Ayuso, J. (2006). Profesión docente y estrés laboral: una aprximación a los conceptos de estrés laboral y burnout. Revista Iberoamericana de Educación, $1-15$.

Botero Álvarez, C. (2012). Riesgo psicosocial intralaboral y burnout en docentes universitarios en algunos países latinoamericanos. Cuadernos de Administración. Universidad del Valle, 2-16.

Caballero, C., Hederich, C., \& Palacio, J. (2010). El burnout académico: delimitación del síndrome y factores asociados con su naparición. revista Latinoamericana de Psicología, 131-146.

Cabezas, E. (2016). Análisis del síndrome de burnout en docentes de la facultad de Ingeniería de la universidad Nacional de Chimborazo y su incidencia en el desempeño laboral . Industrial Data, 59-68.

Cárdenas, M., Méndez, L., \& González, M. (2014). Evaluación del desempeño docente, estrés y burnout en profesores universitarios. Industrial Data, 1-22.

Carlotto, M., \& Camara Goncalves, S. (junio de 2017). Riscos psissociais associados a sindrome burn out en professores universitarios. Avances en Psicología Latinoamericana, 53(3).

Carlotto, M., \& Goncalves Camara, S. (abril de 2007). Preditores de sindrome burnout em professores. Revista de Psicología Escolar e educacional, 11(1).

Chavarría, R., Colunga, F., \& Peláez, K. (2017). Sindrome burnout en médicos docentes de un hospital en segundo nivel en México. Educación Médica, 254-261.

Chávez, C. (2016). Síndrome de Burnout en docentes universitarios. Research Journal, 77-95.

Díaz Bambulla, F., \& Gómez, I. (2016). La investigación sobre el síndrome burnout en latinoamericana entre 2000 y 2010. Psciología desde el Caribe, 113-131.

Díaz, A. (23 de julio de 2021). Influencia del sín-

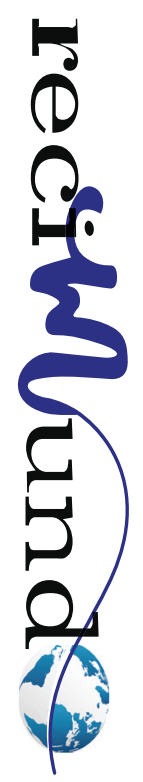


drome Burnout en el desempeño docente de los profesores de la facultad de Ciencias de la Salud de la Universidad Nacional del Callao. Obtenido de www.eumed.net: https://www.eumed.net/rev/ caribe/2018/03/estres-docentes-universitarios. html/hdl.handle.net/20.500.11763/caribe1803estres-docentes-universitarios

Gil Monte, P. (abril de 2011). El síndrome de quemarse por el trabajo (síndrome de burn out): aproximaciones teóricas para su explicación y recomendaciones para la intervención. Revista de psicología científica, 3(5).

Guyton, Y., \& Hall, J. (2016). Tratado de fisiología médica. México: Elsevier.

Hernández Sampieri, R. (2014.). Metodología de la investigación. Sexta edición. México: McGraw Hill.

Jonsdettir, F., \& Dahlman Sjors, A. (2018). Endocrine and inmunology aspects of burnout: a narrative review. International Journal of Endochrinology.

Lou, G., \& Chen, Y. (2016). A Study of the English teachers' burnout in a local comprehensible University in China. Creative Education, 646-654.

Merces, M., Almeida Lopes, R., Souza e Silva, D., \& Souza Oliveira, D. (2017). prevalencia da sindorme burnout em profissionais de emfermagem da atencao basica a saude . Revista de Pesquisa Cuidado e Fundamental Online, DOI 109789/21755361.2017.V9I.208-214.

Mora, J., Flores, Y., Hernández, V., Sánchez, E., \& Marroquin, R. (2015). Parámetros biológicos del burnout en docentes de la carrera. México.

Morales, S., \& Hidalgo Murillo, L. (Marzo de 2015). Síndorme de Burnout. Una revisión bibliográfica. Medicina Legal de Costa Rica, 31(1).

Mouzo Quintans, J. (2019). Recuperado el 7 de abril de 2021, de El "bournout" toma peso en la lista de dolencias de la OMS: https://elpais.com/sociedad/2019/05/27/actualidad/1558956228_933147. html

Olivares, V. (2017). Laudatio doctora Christina Maslach. comprendiendo el Burnout. Revista Ciencia y Trabajo, 59-63.

Organización Internacional del Trabajo. (2020). El trabajo de cuidados y los trabajadores de cuidado. Para un futuro con trabajo decente. ejecutivo, Organización Internacional del Trabajo, Trabajo decente, Nueva York.

Pedro, M. (2001). El síndrome de quemarse en el trabajo (síndrome de nurnout): aproximaciones teóricas para su explicación y recomendaciones para la intervención. Revista Psicología Científica, 3-7.

Pérez Jauregui, M. (2018). Síndrome de burnout, contexto social y subjetividad. Revista de Ciencias Empresariales y Sociales.

Pineda Valladares, M., Rivera Rodas, M., \& Ríos Verdugo, P. (2014). Prevalencia y factores influyentes del sindrome burnout en los docentes de la Facultad de Ciencias Médicas de la Universidad de Cuenca (primera ed.). (U. d. Cuenca, Ed.) Cuenca, Ecuador: Universidad de Cuenca.

Ramírez, M. (2017). Prevalencia del síndrome Burnout y la asociación con variables sociodemográficas y laborales en una provincia de Ecuador. International Journal of Development and Educational Psychology, 241-252.

República del Ecuador. (2016). Ley Orgánica de Educación. Quito: República del Ecuador.

Rodríguez, L., bermello, I., Pinargote, E., \& Durán, U. (2018). El estrés y su impacto en la salud mental de los docentes universitarios. Revista Caribeña de Ciencias Sociales, 35-42.

Rozas Caamaño, G. (2016). Niveles de desgaste profesional (burnout) en docentes y estrategias de prevención. El caso de las facultades de salud, Educación y Ciencias Sociales de la Universidad de Santo Tomás. Málaga: Servicio de Publiaciones y Divulgación Cientifica.

Santana Cárdenas, S. (2016). Relationship of work stress with eating behavior and obesity: Theoreticaland empirical considerations. Revista Mexicana de Trastornos Alimentarios, 135-143.

Serrano, R. (2006). Adaptación psicobiológica al estrés social en una muestra de profesores: cambios hormonales, cardiovasculares y psicológicos. VaIencia: Universitat de Valencia.

Sierra Bravo, R. (1994). Métodos y Técnicas de Investigación Social. Caracas: Facultad de Humanidades UCV. 
CREATIVE COMMONS RECONOCIMIENTO-NOCOMERCIAL-COMPARTIRIGUAL 4.0.

\section{CITAR ESTE ARTICULO:}

Gurumendi España, I. E., Patricia Panunzio, A., Calle Gómez, M. A., \& Borja Santillán, M. A. (2021). Síndrome burnout en docentes universitarios. RECIMUNDO, 5(3), 205-219. https://doi.org/10.26820/recimundo/5.(2).julio.2021.205-219 